INPLASY

PROTOCOL

To cite: Shao et al. Efficacy and Safety of Emergent Transcatheter Aortic Valve Implantation in Patients with Decompensated Aortic Stenosis. Inplasy protocol 202140050. doi: 10.37766/inplasy2021.4.0050

Received: 07 April 2021

Published: 08 April 2021

Corresponding author: Shao Ruochen

1054669904@qq.com

Author Affiliation:

Sichuan University

Support: Natural Science

Foundation.

Review Stage at time of this submission: Preliminary searches.

Conflicts of interest:

None declared.

\section{Efficacy and Safety of Emergent Transcatheter Aortic Valve Implantation in Patients with Decompensated Aortic Stenosis}

Shao, RC1; Li, JL².

Review question / Objective: According to the current guidelines for the treatment of valvular diseases, hemodynamic instability is the relative contraindication of TAVR. Most TAVR is performed selectively in patients with hemodynamic stability. However, more and more studies have shown that emergent TAVR may be feasible and effective in severe as patients with cardiogenic shock or acute decompensated heart failure. Therefore, we performed a systematic review and meta-analysis to comprehensively and quantitatively investigate the efficacy and safety of emergent TAVR compared with elective TAVR, to provide evidence for clinical treatment decision-making.

Condition being studied: Decompensated Aortic Stenosis.

INPLASY registration number: This protocol was registered with the International Platform of Registered Systematic Review and Meta-Analysis Protocols (INPLASY) on 08 April 2021 and was last updated on 08 April 2021 (registration number INPLASY202140050).

\section{INTRODUCTION}

Review question / Objective: According to the current guidelines for the treatment of valvular diseases, hemodynamic instability is the relative contraindication of TAVR. Most TAVR is performed selectively in patients with hemodynamic stability. However, more and more studies have shown that emergent TAVR may be feasible and effective in severe as patients with cardiogenic shock or acute decompensated heart failure. Therefore, we performed a systematic review and meta-analysis to comprehensively and quantitatively investigate the efficacy and safety of emergent TAVR compared with 
elective TAVR, to provide evidence for clinical treatment decision-making.

Condition being studied: Decompensated Aortic Stenosis.

\section{METHODS}

Participant or population: Patients with Decompensated Aortic Stenosis.

Intervention: Emergent Transcatheter Aortic Valve Implantation.

Comparator: Elective Transcatheter Aortic Valve Implantation.

Study designs to be included: Randomized clinical trials and prospective/retrospective cohort studies.

Eligibility criteria: (1)randomized clinical trials and prospective/retrospective cohort studies; (2)reported Outcome indicators for both emergent TAVR and emergent TAVR;

(3) reported the specific number or incidence of outcome indicators or displayed the survival curve.

Information sources: PubMed, Embase and The Cochrane Library.

Main outcome(s): Post-TAVI complications, in-hospital mortality and 30 days and 1 year mortality after TAVI.

Quality assessment / Risk of bias analysis: The Newcastle-Ottawa Scale (NOS) was used to assess the quality of the included observational studies.

Strategy of data synthesis: Revman $\mathbf{5 . 3}$ software was used for statistical analysis. The relative risk (RR) was used as the statistic of effect analysis, and the $95 \% \mathrm{Cl}$ of each effect was given. The heterogeneity of the included results was analyzed by chi square test (the test level was $a=0.1$ ), and the heterogeneity was quantified by combining with 12 . If there was no statistical heterogeneity among the results, the fixed effect model was used for metaanalysis; if there was statistical heterogeneity among the results, the sources of heterogeneity were further analyzed. After excluding the influence of obvious clinical heterogeneity, the random effect model was used for meta-analysis. Significant clinical heterogeneity was treated by subgroup analysis or sensitivity analysis, or only descriptive analysis. The level of meta analysis was set as $\mathbf{a}=\mathbf{0 . 0 5}$.

Subgroup analysis: According to the degree of emergency, it can be divided into Urgent/emergent/elective TAVI.

Sensitivity analysis: We performed sensitivity analysis by discarding one study at a time and repeating the meta-analysis to examine the robustness of the pooled results.

\section{Language: English.}

Country(ies) involved: China, USA, UK, Canada.

Keywords: aortic stenosis, transcatheter aortic valve implantation, emergent procedure.

Contributions of each author:

Author 1 - Shao Ruochen.

Author 2 - Li Junli. 